\title{
An Eco-friendly Hybrid Urban Computing Network Combining Community-based Wireless LAN Access and Wireless Sensor Networking
}

\author{
Maarten Wijnants* ${ }^{*}$, Wim Lamotte*, Nicolas Letor ${ }^{\dagger}$, Chris Blondia ${ }^{\dagger}$, Eli De Poorter ${ }^{\ddagger}$, Dries Naudts ${ }^{\ddagger}$, Stijn Verstichel ${ }^{\ddagger}$, \\ Bart Lannoo $^{\ddagger}$, Ingrid Moerman ${ }^{\ddagger}$, Nelson Matthys ${ }^{\S}$, Christophe Huygens ${ }^{\S}$ \\ ${ }^{*}$ Hasselt University - $t U L-I B B T-$ Expertise Centre for Digital Media \\ Wetenschapspark 2, 3590 Diepenbeek, Belgium - \{firstname. Iastname $\}$ duhasselt.be \\ †University of Antwerp - IBBT - PATS \\ Middelheimlaan 1, 2020 Antwerpen, Belgium - \{firstname. lastname $\}$ eua.ac.be \\ ${ }^{\ddagger}$ Department of Information Technology - IBBT - Ghent University \\ Gaston Crommenlaan 8 bus 201, 9050 Ghent, Belgium - \{firstname. lastname\} @intec.ugent.be \\ ${ }^{\S}$ IBBT-DistriNet, Department of Computer Science \\ KU Leuven, 3001 Heverlee, Belgium - \{firstname. Iastname\} @es.kuleuven.be
}

\begin{abstract}
Computer-enhanced smart environments, distributed environmental monitoring, wireless communication, energy conservation and sustainable technologies, ubiquitous access to Internet-located data and services, user mobility and innovation as a tool for service differentiation are all significant contemporary research subjects and societal developments. This position paper presents the design of a hybrid municipal network infrastructure that, to a lesser or greater degree, incorporates aspects from each of these topics by integrating a community-based Wi-Fi access network with Wireless Sensor Network (WSN) functionality. The former component provides free wireless Internet connectivity by harvesting the Internet subscriptions of city inhabitants. To minimize session interruptions for mobile clients, this subsystem incorporates technology that achieves (near-)seamless handover between WiFi access points. The WSN component on the other hand renders it feasible to sense physical properties and to realize the Internet of Things (IoT) paradigm. This in turn scaffolds the development of value-added end-user applications that are consumable through the community-powered access network. The WSN subsystem invests substantially in ecological considerations by means of a green distributed reasoning framework and sensor middleware that collaboratively aim to minimize the network's global energy consumption. Via the discussion of two illustrative applications that are currently being developed as part of a concrete smart city deployment, we offer a taste of the myriad of innovative digital services in an extensive spectrum of application domains that is unlocked by the proposed platform.
\end{abstract}

Keywords-Urban computing, smart spaces, sensor, wireless sensor network, community-based Internet access, seamless Wi-Fi connectivity, energy efficiency, digital service innovation

\section{INTRODUCTION}

The pervasiveness of networked computing devices in real-life setups is progressively giving rise to computerenhanced physical spaces of divergent dimensions. An example of setups of moderate size are computer-enhanced smart meeting rooms in buildings, while urban computing setups are good illustrations of large-scale ubiquitous systems. The urban computing philosophy revolves around the integration of technology and computational power in public environments such as cities and parks. Closely related to this ubiquitous computing paradigm is the Internet of Things (IoT) concept where, via the use of technology, real-world objects become uniquely identifiable and form a network of Internet-enabled entities [1]. As is the case with pervasive deployments, (low-cost) distributed Wireless Sensor Networks (WSNs) that are able to observe and sense specific contextual and environmental features typically play a primordial role in the operation of IoT configurations. Combined, the mentioned technologies hold promising potential to satisfy people's continuously burgeoning desires and requirements with regard to ubiquitous information access and digital service consumption. At the same time, they scaffold the development of heterogeneous types of innovative end-user applications that range from purely informative offerings (e.g., reporting environmental measurements) to true value-added services (e.g., digital tools that support, facilitate or accelerate the execution of a particular human task).

Another important observation is that societal attention for environmentalism and ecological sustainability is nearly becoming common practice. The heightened consciousness of politicians, city administrators and people in general regarding the need to minimize the ecological impact of products and services manifests itself in divergent application domains, including computing and network infrastructure. Consequently, it is advisable for ubiquitous deployments and WSNs to aspire a minimal environmental footprint by making conservative use of energy and potentially also other scarce (natural) resources.

In this work-in-progress report, we describe our ongoing efforts concerning the design and realization of an energyaware dual urban computing and networking environment. Its duality lies in the fact that the infrastructure encompasses 
both a community-powered wireless Internet offering for end-users and WSN deployments. City-scale access to digital information and Internet-based services is guaranteed via the concept of Wi-Fi crowdsourcing. At the same time, the WSN subsytem enables the dynamic aggregation of relevant environmental measurements as well as real-time status tracking of physical objects. To the best of our knowledge, no similar urban computing solutions have been proposed that simultaneously address wireless Internet connectivity and sensor-based monitoring and information accumulation. A further distinguishing feature of our platform are its eco-friendly aspirations: an incorporated green distributed reasoning framework and sensor middleware measures cooperate to minimize the energy expenditure of the WSN branch of the system.

The remainder of this paper is organized as follows. Section II presents the global methodology and high-level architectural design of the proposed urban networking system. Next, detailed descriptions regarding each of the infrastructure's constituting subsystems will be provided in Section III. Section IV outlines our plans for a Proof-of-Concept (PoC) deployment in a Belgian city. The PoC demonstration will include two example digital urban services, whose development is currently ongoing and is treated in Section V. Finally, Section VI briefly reviews related work, while Section VII draws our conclusions.

\section{System Design}

The proposed system's multi-faceted nature yields an endto-end solution whose operational chain spans from data registration at the individual IoT node level all the way up to end-user service delivery and sensed data consumption via the included community-based wireless access network. The architectural design, shown in Figure 1, effectively combines multiple WSNs, a community Wi-Fi network, a back-end server LAN and an intelligent "green" middleware into an integrated urban computing environment.

The WSNs are responsible for capturing information about the physical environment and for transmitting the resulting data to the back-end servers. Sensor nodes connect to the back-office via a sensor gateway, hereby optionally multi-hopping over intermediate sensor routers. The WSNs are equipped with a limited "green intelligence", which locally directs the energy consumption of its composing sensor nodes in order to improve their battery lifetime.

The back-end servers provide persistent storage for information observed by the WSNs, potentially process this data and make it accessible for authorized external parties through a relational database. In addition, because the backend has a comprehensive overview of the complete WSN organization, the servers host the coordinator of the distributed sensor reasoning framework and the green WSN middleware that optimizes power efficiency across all involved WSNs

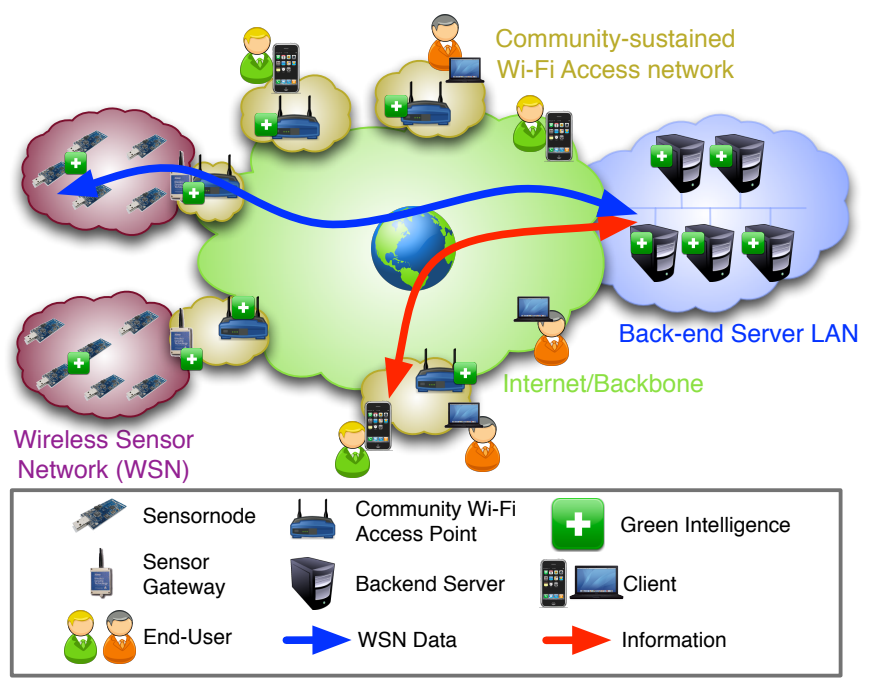

Figure 1. High-level schema of the hybrid system architecture

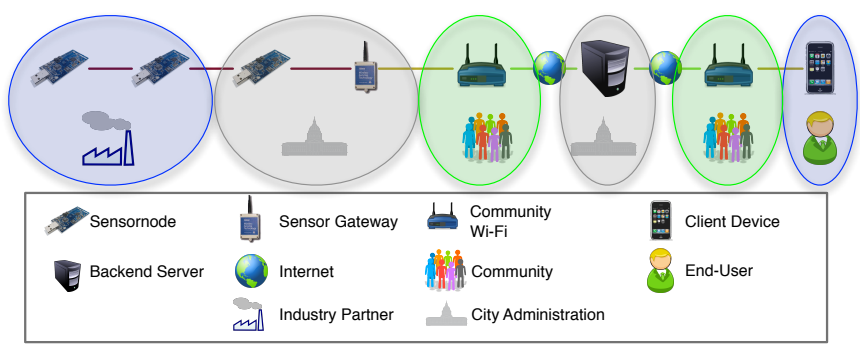

Figure 2. Network hardware ownership

simultaneously, this way effectively contributing to the ecofriendliness of the wireless sensing environment.

The city-wide community-sustained Wi-Fi network fulfills a dual role by simultaneously acting as an uplink for the WSNs and providing end-users with Internet access. As such, it caters to the expanding end-user demand for ubiquitous Internet connectivity and it enables users to access the information stored in the back-end. The Wi-Fi subsystem consists of a collection of wireless access points (APs), which are owned by volunteer citizens or governmental agencies and which are deployed at their premises. An enduser connects to a community AP with a Wi-Fi-enabled terminal (e.g., a smartphone or tablet PC) without being required to install any specialized software. The community network technology contains mobility solutions, allowing the end-user to freely roam the environment while consuming information.

The proposed two-dimensional network infrastructure envelops diverse types of hardware components. The ownership and responsibility for each of these entities is allocated to involved stakeholders as is illustrated in Figure 2:

- Each WSN is jointly operated by industrial parties and the city administration. The municipality is mandated to deploy WSN relay infrastructure that can be shared 


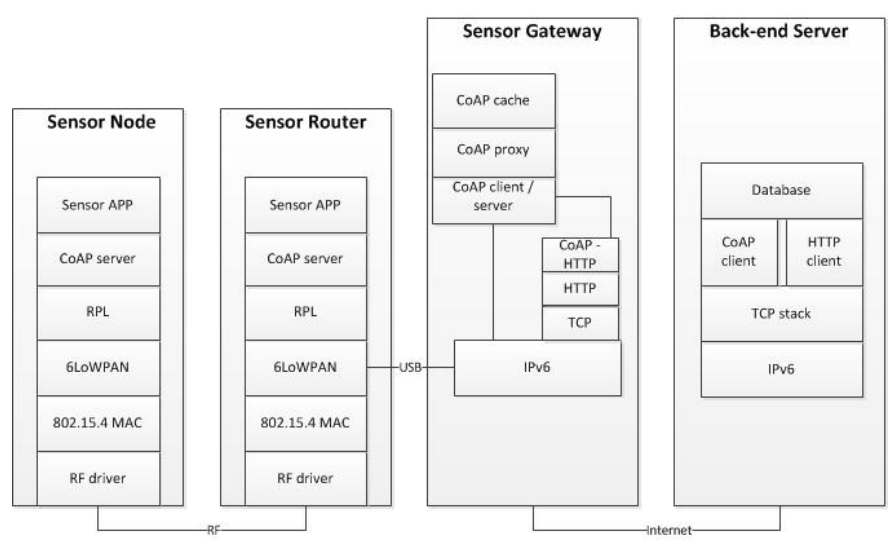

Figure 3. WSN architecture and involved device typology

by multiple industrial partners, each deploying their proprietary low-power sensors in the field. An example scenario consists of the city administration installing relay sensors in lampposts to create a non-powerconstrained WSN backbone. The sensor gateway either connects to the community network as a Wi-Fi client, or is directly integrated in the community access point.

- Community access points are property of the citizens, which volunteer to share their Internet connection.

- The back-end servers are typically placed under the administrative authority of the city council. Because sensed data is collected over a geographically vast area, it is advisable to store, guard and process such information under supervision of a governmental body. The system design imposes that all information inquiries pass through the back-end for security checking.

- End-user devices connecting via the community network are evidently owned by the citizen or city visitor.

\section{Composing Technologies And Features}

\section{A. WSN Architecture}

A schematic overview of the WSN architecture is given in Figure 3. As is apparent from the diagram, heterogeneous types of devices are involved in the WSN subsystem:

- Sensor nodes obtain information pertaining to the environment and track the status of physical attributes and objects. Power-wise, they are either battery-driven or rely on energy-scavenging methods (e.g., solar power). To limit their complexity and energy consumption, sensor nodes lack routing functionality.

- Sensor routers provide multi-hop capabilities to the sensor network. Data coming from the sensor nodes is routed towards the sensor gateway using the IPv6 Routing Protocol for Low-Power and Lossy Networks (RPL) [2]. The network is built in a hierarchical manner, with sensor routers assuming the role of intermediary forwarding nodes. The sensor routers form a tree-like topology that is rooted at the sensor gateway.

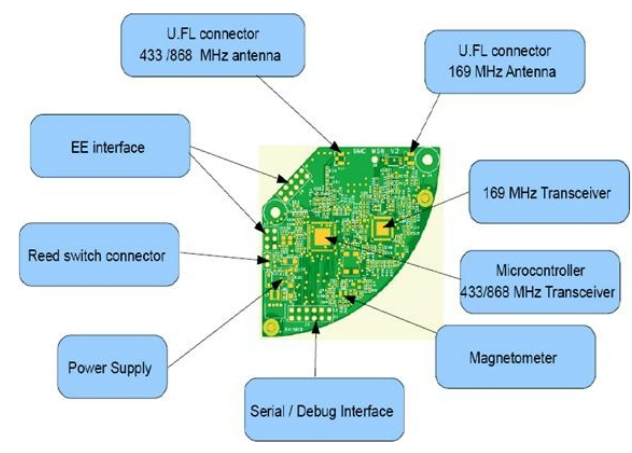

Figure 4. Sensor node hardware design

Routers may or may not be outfitted with a sensing device and are typically permanently powered on.

- The sensor gateway serves as an uplink for the wireless sensors. By connecting to the community-based Wi-Fi network (see Section III-C), the gateway gains Internet access.

In terms of communication, all WSN nodes employ standardized IEEE and IETF network protocols. For the physical and MAC layer, the IEEE 802.15.4 standard [3] is used, which is designed for low-speed ubiquitous communication between low-cost devices. To cope with the size limitations of 802.15.4 packets, the WSN architecture adopts the 6LoWPAN adaptation layer [4], which includes encapsulation and header compression mechanisms (RFC 6282) to reduce the size of the IPv6 packets. Sensor information is transported to the back-end servers by means of the IETF Constrained Application Protocol (CoAP) [5], a specialized RESTful web transfer protocol that has recently been standardized for machine-to-machine communication involving constrained networks and nodes. A CoAP proxy [6] is installed on the sensor gateway that either forwards the CoAP messages to the back-end in the form of IPv6 packets, or translates the CoAP data to HTTP as a fallback solution for communication over the classic Internet infrastructure.

\section{B. Sensor Design and Hardware}

The board layout of the sensor devices is shown in Figure 4. The sensor devices use a Cortex M3 micro controller (ADUCRF101) with on chip 128K flash memory and $16 \mathrm{~K}$ ram. The radio operates in the $169.4-169.8$ $\mathrm{MHz}$ frequency band. This frequency range was recently reallocated [7] by the European Commission (Commission Decision 2008/673/EC) to open up a host of new applications, including meter reading systems for value-added consumer services. Compared to the $2.4 \mathrm{GHz}$ or $868 \mathrm{MHz}$ frequencies that are traditionally used for sensor networks, this low frequency band is far less hindered by obstacles (such as parked cars or buildings) and bad weather effects. As a result, less dynamic link failures occur and the overall transmission range is significantly boosted (up to several 


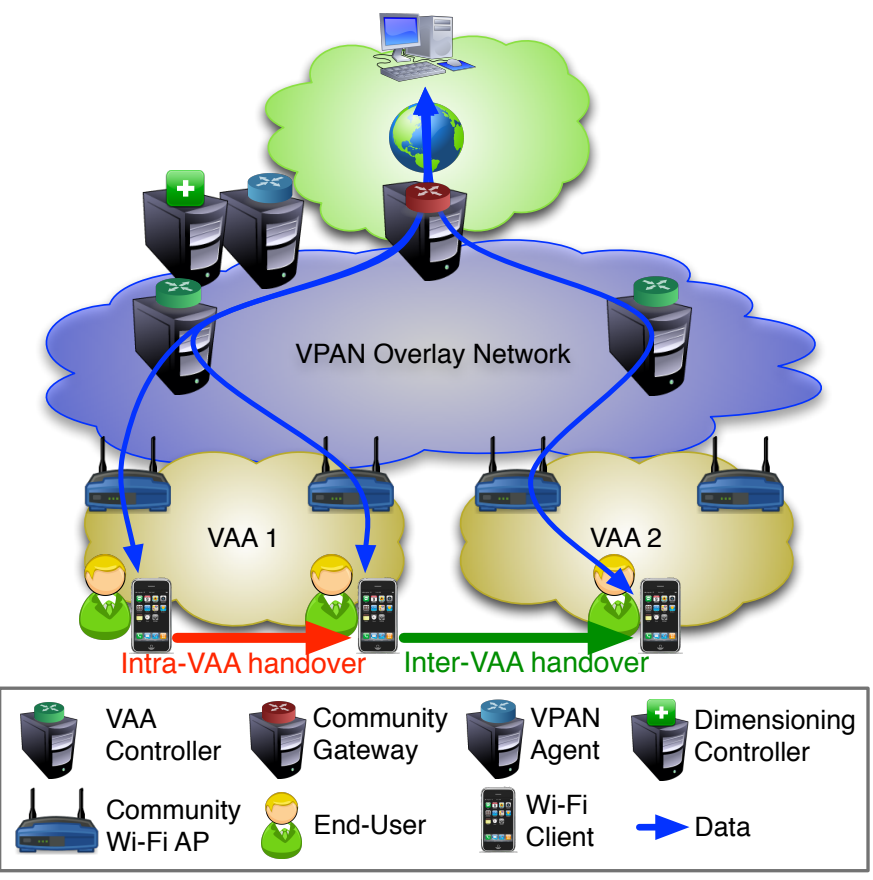

Figure 5. Architecture of the community-based Wi-Fi access network and the Virtual Access Area concept

kilometers in rural areas). To guarantee backward compatibility with legacy sensor nodes, a second RF transceiver is installed that supports the 433 or $868 \mathrm{MHz}$ band.

\section{Community-Based Wi-Fi Access}

The urban-scale community-powered Wi-Fi subsystem is modeled as a two-tiered networking platform, whose architecture is illustrated in Figure 5. The base layer is populated by individual Wi-Fi access points that are dispersed over the territory of the city by encouraging residents to share their wireless Internet connection with the community, in a fashion similar to the Fon Wi-Fi crowdsourcing and community networking approach [8]. These so-called community APs (CAPs) are geographically clustered into multiple Virtual Access Areas (VAAs) that share the same network SSID. A VAA enables seamless session handover between its constituting WLAN APs by behaving as a single Wi-Fi access point with a significantly augmented transmission range. Client stations are unaware of occurring intra-VAA handovers, because all CAPs that are member of a single VAA exhibit identical operating characteristics (SSID, BSSID and operating channel). When crossing a VAA boundary, an inter-VAA handover that is not seamless yet transparent is initiated by a back-end controller. In particular, the user may perceive a slight service interruption in this process, but any ongoing communication will resume swiftly, with preservation of the client-side network address. The second tier of the model provides a Virtual Private Ad Hoc Network (VPAN) overlay [9] that connects the individual CAPs with the back-end servers, which in turn implement the management of the community network (including handover orchestration). The VPAN system provides a secure and self-organizing virtual overlay network that spans separate (groups of) nodes by employing ad hoc network techniques and a private addressing space, and by securing communication between these nodes.

The back-end operational coordination of the community Wi-Fi network encompasses the following functional entities:

- The VAA Controller is responsible for the coordination of a single VAA and for handling seamless client handovers from one CAP to another within that VAA. It collects signal statistics from all its constituting CAPs and assigns the client device to an optimal access point for servicing. In addition, a VAA Controller is liable for forwarding incoming traffic to the CAP that is servicing the destined terminal and, conversely, for propagating outbound traffic to its destination.

- The Community Gateway acts as an inter-VAA handover coordinator and as a network anchor point. Inbound (Internet) traffic as well as local traffic are forwarded to the appropriate VAA Controller. In addition, the gateway checks egress traffic for authorization. If necessary, the traffic session is captured and redirected to a web portal like NoCat [10] [11] for authentication. Once the user is authenticated, access to the requested information will be granted.

- The Dimensioning Controller optimizes the coverage of the multiple VAAs in order to cope with roaming, fluctuating traffic volumes and dynamic end-user mobility patterns. If changes are required, the Dimensioning Controller retasks the community access points with a new configuration, this way effectively reorganizing the VAAs.

- The VPAN Agent serves as a directory service. VPAN nodes must register themselves with this agent, which subsequently aids them with the discovery of other nodes and with the establishment of secure tunnels between distant VPAN nodes.

\section{Green Intelligence for Environment-Conscious WSNs}

The proposed network architecture hosts a two-fold environmentally aware intelligence that combines a distributed sensor reasoning framework with a sensor middleware which jointly strive to energy-wise maximize WSN sustainability.

1) Distributed Sensor Reasoning Framework: This process configures the sensor nodes and their parameters so that their operation becomes more eco-friendly, primarily in terms of energy expenditure. The reasoning logic is implemented at two separate tiers, namely on individual sensor nodes as well as on the coordinating back-end. Due to the limited processing power of the sensors, their reasoning capabilities are rather limited. The aim here is to determine 
a local energy consumption optimum through direct manipulation of proprietary low-level attributes. On the other hand, the back-end management entity has a holistic overview of the status of every WSN and hence is able to calculate and enforce a global optimum for each WSN as a whole. Potential configuration parameters that could be influenced by the reasoning framework include polling schemes (e.g., polling frequency), sensor data poll versus sensor data push, sleeping strategies, sensor node transmission power, etcetera.

The reasoning framework is founded on a generic domain model. The preferred approach to define such a model is through the adoption of ontology-based technologies [12]. For the proposed urban network, the model for the sensors and the WSN subsystem has been designed as an extension of the Semantic Sensor Network Ontology, developed by the W3C Semantic Sensor Network Incubator Group [13]. The D2R engine [14] hereby fulfills the role of ontology data provider (by mapping data that is available in the application framework's relational database, see Section III-E). It is clear from the start however that an ontology-based solution, supported only by traditional description logics reasoning algorithms [15], will neither be sufficient nor feasible, for example due to the resource-constrained character of WSN setups [16]. Therefore, in addition to reasoning techniques based on traditional description logics to determine the global optimum by the back-end coordinator, automatic code generation procedures are adopted to generate sensor node reasoning components which are deployed and activated on the individual sensor nodes to determine their local optimum in terms of power efficiency. The model should therefore be seen as a common understanding, from which the adopted application mechanisms can derive the proprietary representation.

2) Component and Policy Infrastructure (CaPI): $\mathrm{CaPI}$ is a green WSN middleware prototype that features the combination of a component-based runtime and a policy-driven management framework [17]. Software components in CaPI provide coarse-grained, service-like units of functionality. Components hence represent binary blobs of code and are used to implement basic application objectives, like sensing or data processing. In contrast, policies are of a much more fine-grained nature and encapsulate specific rules to govern the runtime behavior of the sensor. Policies are lightweight scripts that may contain the configuration settings related to the energy-efficient operation of components. Furthermore, policies may envelop some simple application objectives as well, such as filtering or intelligent aggregation, this way allowing for cost-effective reconfiguration and reorientation of the WSN software over time. The dynamic deployment of CaPI components and policies is controlled by the backend to enact advocated changes to the software setup of WSN nodes and to enforce the greenest possible sensor configuration. The integration of programming paradigms of such diverse orders of granularity yields significant flex-

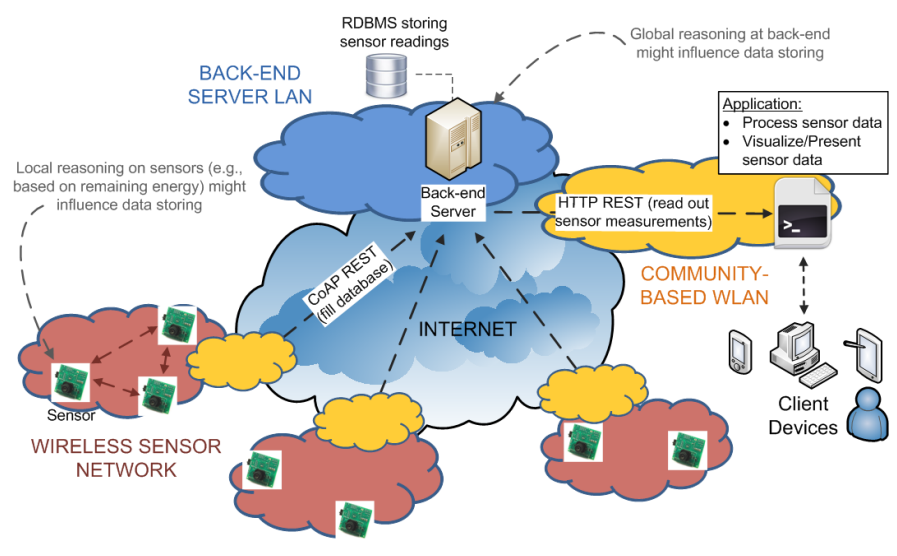

Figure 6. Conceptual overview of the application framework

ibility, removes the need for expensive replacements of a sensor node's integral software image, and might result in meaningful energy and operational savings with regard to (re)configuration costs.

\section{E. Application Framework}

An application framework is provided to support the development of services and applications that base their operation on accumulated sensor observations. By applying a separation of concerns design, the framework enforces a clean and complete division between the sensor networks and the application logic by enacting a back-end relational database as a go-between. As such, it suffices for IoT nodes to store (via CoAP) their measurements in the relational database that is hosted in the back-office. Applications on the other hand are provided a HTTP-based RESTful interface to query this database and to obtain up-to-date sensed information. This design allows the WSN architecture and the application framework to evolve largely independently from each other. Moreover, it significantly simplifies the necessary security mechanisms as it allows all security-related operations to be centrally enforced. Protocol translation provisions between IPv6-based sensor networks and clients' Internet traffic, which generally still employs the IPv4 protocol, are also rendered obsolete. Finally, the relatively compact HTTP interface results in a fairly declarative programming model, which in turn is expected to expedite the development of end-user urban services.

Besides bare sensor observations, the back-end database maintains two other conceptual categories of information. The first consists of prevailing hardware-, software- and network-related characteristics of the WSN subsystem, such as a WSN node's remaining battery power or wireless signal strength. This type of knowledge is fed to the green sensor intelligence so that it can derive well-informed WSN configuration instructions (see Section III-D). Secondly, the database captures service-dependent information that supports, embellishes or even enables application execution. It is apparent that such content can be highly heterogeneous. 
For the envisioned personal parking assistant application (which will be described in Section V-A), it for instance encompasses the geospatial dimensions of tracked parking locations and applicable parking fees.

Another responsibility that is bestowed upon the database is sensor failure tracking. Malfunctioning or defect nodes are a tangible reality, especially in public WSN deployments. This is partly due to the fact that sensors are liable to wear (e.g., battery depletion, hardware defects), but also vandalism is a probable cause for this phenomenon. To guarantee the operational continuity of the urban computing network and the services it offers to end-users, failed sensors need to be replaced. The database implements the management and administration of such sensor replacement operations.

\section{Proof-OF-CONCEPT DePloyment}

In the next few months, a comprehensive Proof-ofConcept realization of the proposed urban computing setup will be rolled out in Ghent, a Belgian city with a population of approximately 250.000 people. The deployment will encompass around 50 sensor nodes that initially will concentrate on the monitoring of 2 distinct physical properties: parking spaces and garbage bins. Depending on the involved property, different sensors are mounted on the sensor board. The presence of a car on a parking spot is detected by measuring the distortion of the earth's magnetic field in all three spatial axes. To facilitate the practical deployment of this type of sensor in the city, the sensor board layout has been designed to fit within a commercially available circular "speeddisk" (a device for slowing down traffic), which is subsequently mounted with a screw on the parking spot at street level (see Figure 7(a)). For the garbage bins on the other hand, the opening and closing of their lid is monitored by a magnet-activated reed switch (see Figure 7(b)). Furthermore, at least 10 community access points will be included in the deployment. These will be divided into 2 or 3 VAAs, which combined will cover a region of roughly 2 square kilometres. Besides to empirically confirm the technological validity of our approach, the $\mathrm{PoC}$ installation will serve to evaluate the qualitative features of the network's two-fold design and of its envisaged service offerings (see Section V). Appropriate user experience research mechanisms will be applied to conduct the qualitative assessment.

\section{Example URban SeRvices}

\section{A. Personal Parking Assistant (PPA)}

Car parking in urban areas forms an important challenge in present-day society. A real-time Personal Parking Assistant (PPA) application for smart city environments, which dynamically visualizes on a hand-held device vacancy information pertaining to parking locations near a certain destination, is a promising tool to alleviate the burden of searching for an available parking spot. As such, such a service yields direct revenue for end-users since they will
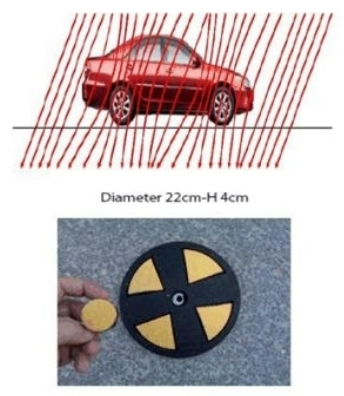

(a)

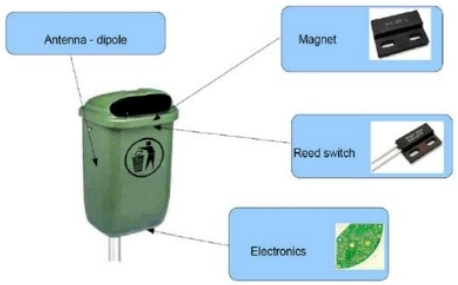

(b)
Figure 7. Sensor deployment and sensed physical properties: (a) personal parking assistance; (b) garbage bin tampering detection

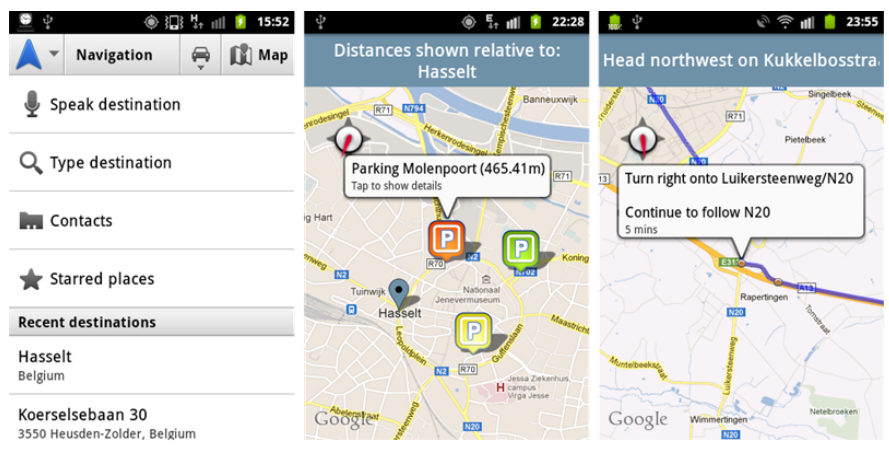

Figure 8. Tentative Personal Parking Assistant mock-ups

witness an improvement of their urban life. At the same time, the municipality stakeholder will likely indirectly also benefit in the sense that offering outdoor parking control services is expected to positively impact people's perception and opinion regarding the commuting options within the city. The city administration moreover gains valuable insight in the parking patterns within their territory and could exert this data in future urban planning initiatives.

To realize the PPA use case, a limited amount of on-street parking locations will be monitored by a sensor node (see Figure 7(a)). The on-street parking measurements are complemented with externally provided dynamic data pertaining to the occupancy rate of off-street parking garages. Finally, besides availability information, other parking-related data such as the applicable fee, spatial dimensions of the parking spot, hourly parking limits, paying modes and reservation options will also be integrated in the service.

\section{B. Garbage Bin Tampering Monitor (GBTM)}

In the city of Ghent, a number of public garbage bins are installed that are intended exclusively for small-size litter disposal via a hole in their lid. To empty this type of dustbin, their cover needs to be removed, an operation that is strictly reserved for municipal employees. In the GBTM use case, a selected portion of this dustbin category will be instrumented with a sensor that will record the opening and closing of their cover (see Figure 7(b)). The resulting measurements, including their time of occurrence, are stored in the back-end 
database. Via a web-based interface, the municipal garbage authority is able to consult the registered information.

The essential objective of the GBTM application is to facilitate the detection of illegitimate activities concerning public garbage bins. Examples of such activities include vandalism and illegal waste disposal (i.e., unlawfully removing the cover to be able to dump voluminous objects). By crossreferencing the sensed information and their timestamp with the city's garbage collection schedules, the qualified agency will be able to swiftly identify potential criminal offenses and to react appropriately. A secondary advantage of the service is that it might assist the municipality in optimizing garbage collection routes and timetables by revealing potential issues or lacunae in the schedule. The service also exhibits a high relatedness to the "green" mindset of the proposed bipartite network.

\section{RELATED WORK}

Urban-scale WLANs have been rolled out in a number of cities over the past few years. Examples include Minneapolis, Montreal, Leiden and Bruges [18]. Usually a commercial network operator closely collaborates with the local government to set up the municipal network and acts as a wireless Internet service provider. More recently, wireless community-sustained networks have proven to offer a viable alternative to such business-coordinated initiatives [19] [20]. The Meraki project [21] in San Francisco and the Fon project [8] are very promising approaches in this context. The Belgian telecom operator Belgacom has recently initiated a partnership with Fon, this way allowing its customers to surf via Fon hotspots worldwide [22]. Telenet, another Belgian operator, is currently in the process of deploying an analogous yet proprietary Wi-Fi crowdsourcing solution [23].

Seamless Wi-Fi connectivity for mobile users is still not a reality. For IEEE 802.11, a multitude of handover mechanisms have been investigated, the majority of which introduce ways to optimize the three main 802.11 handoff phases [24]-[28]. Others have proposed a fast handoff scheme that is controlled and prepared by the access network [29]. A salient drawback of this latter solution is that a small dedicated component must be installed on the client device. In contrast, the wireless access network that is included in our network infrastructure ensures fast handover without necessitating the deployment of special-purpose software at client side. The methodology of the VAA concept eliminates the need to perform scanning, authentication and reassociation when switching between physical APs. The resulting seamless (or at least transparent) Internet connectivity is in addition offered on top of an insecure community-powered city network which, through the use of VPAN technology, is transformed into an integrated secure overlay network.

Matured ubiquitous computing technology has rendered city-wide sensor deployments and the urban computing paradigm pragmatically feasible. A notable effort in this context is CitySense, a wireless networking testbed that aims to provide an open and programmable experimental facility to the research community [30]. The European Community's SmartSantander project [31] pursues a largely comparable objective. As a final example, Zheng et al. take the interesting approach of treating GPS-outfitted taxis as mobile sensor nodes that are capable of representatively recording road-based traffic flows [32]. Contrary to our urban network architecture, none of these real-life city networks include provisions for public Internet access. A further distinguishing feature of our solution is its ecofriendly nature due to the incorporation of a green distributed reasoning framework and sensor middleware that cooperate to minimize the energy footprint of its WSN subsystem.

Although the proposed urban network infrastructure contributes to the state-of-the-art in the research domains from which it adopts technologies, its principal innovation lies in the combination of these technologies into an integrated platform and the powerful options this unlocks. A simple illustration of the beneficial impact of the conceptual and functional duality of our system is given by the fact that its encompassed community-powered access network not only offers wireless Internet access to end-users, but also provides WAN connectivity for the WSNs. As such, setting up dedicated (wireline) connections for this purpose is rendered redundant.

\section{CONCLUSIONS}

Over the last few years, WSN and IoT technology has advanced to such an extent that practical application of these concepts in physical locations of various dimensions has become a tangible reality. In parallel, societal interest in the environment and the efficient exploitation of natural resources is rapidly maturing among end-users and policy makers alike. This position paper has introduced our multi-faceted urban computing infrastructure that integrates wireless sensing functionality with a community-maintained wireless access network. The WSN architecture implements the compilation of real-time measurements pertaining to environmental attributes or real-world objects, whereas the city-scale crowdsourcing of Wi-Fi access points guarantees pervasive Internet access for end-users and as such accommodates ubiquitous client-side information consumption and digital service delivery. The proposed network can be categorized as being environmentally aware in that it includes various measures to minimize the energy consumption of the encompassed WSN components. In addition, technological provisions ensure that roaming end-users in the majority of situations experience seamless wireless connectivity; in the worst-case scenario, only a minimal service interruption is witnessed. Finally, the description of two exemplative urban applications offer a taste of the myriad of innovative and potentially value-added digital services that is enabled by the duality of our setup. 


\section{ACKNOWLEDGMENTS}

Part of this research was performed in the context of the IBBT project GreenWeCan. This project is co-funded by the IBBT (Interdisciplinary institute for Broadband Technology), a research institute founded by the Flemish Government. Companies and organizations involved in the project are ANDROME, Bausch Datacom, Be-Mobile, OneAccess and Stad Gent, with project support of IWT.

\section{REFERENCES}

[1] D. Uckelmann, M. Harrison, and F. E. Michahelles, Architecting the Internet of Things. Springer, 2011.

[2] IETF, "RPL: IPv6 Routing Protocol for Low-Power and Lossy Networks," Online, http://tools.ietf.org/html/rfc6550.

[3] IEEE, "IEEE Standard 802.15.4d," Online, http://standards. ieee.org/getieee802/download/802.15.4d-2009.pdf.

[4] IETF, "IPv6 over Low power WPAN (6LoWPAN)," Online, http://tools.ietf.org/wg/6lowpan/.

[5] IETF, "Constrained Application Protocol (CoAP)," Online, https://datatracker.ietf.org/doc/draft-ietf-core-coap/.

[6] I. Ishaq, J. Hoebeke, J. Rossey, E. De Poorter, I. Moerman, and P. Demeester, "Facilitating Sensor Deployment, Discovery and Resource Access using Embedded Web Services," in Proceedings of the 1st International Workshop on Extending Seamlessly to the Internet of Things (esIoT 2012), Palermo, Italy, July 2012, pp. 717-724.

[7] European Commision, "Final CEPT Report RSCOM04-69 on the Review of the Frequency Band 169.4 - 169.8 MHz," Online, http://ec.europa.eu/information_society/ policy/ecomm/radio_spectrum/_document_storage/mandates/ rsc04_69_ermes.pdf.

[8] Fon, "Freedom to WiFi," Online, http://corp.fon.com/en.

[9] J. Hoebeke, G. Holderbeke, I. Moerman, B. Dhoedt, and P. Demeester, "Virtual Private Ad Hoc Networking," Wireless Personal Communications, vol. 38, no. 1, pp. 125-141, 2006.

[10] NoCat.net, "Infinite Bandwidth Everywhere for Free," Online, http://nocat.net/.

[11] Coova.org, "CoovaChilli - Open Source Captive Portal Access Controller and RADIUS Software," Online, http://www. coova.org/CoovaChilli.

[12] T. R. Gruber, "A Translation Approach to Portable Ontology Specifications," Knowledge Acquisition, vol. 5, no. 2, pp. 199220, June 1993.

[13] P. Barnaghi, M. Compton, O. Corcho, R. G. Castro, J. Graybeal, A. Herzog, K. Janowicz, H. Neuhaus, A. Nikolov, and K. Page, "Semantic Sensor Network XG Final Report," Recommendation REC-rdf-syntax-grammar-20040210, Online, http://www.w3.org/TR/rdf-syntax-grammar/, World Wide Web Consortium, Tech. Rep. XGR-ssn-20110628, June 2011.

[14] C. Bizer and R. Cyganiak, "D2R Server - Publishing Relational Databases on the Semantic Web," Poster presentation at the 5th International Semantic Web Conference (ISWC 2006), Athens, GA, USA, November 2006.

[15] F. Baader, D. Calvanese, D. McGuinness, D. Nardi, and P. Patel-Schneider, The Description Logic Handbook: Theory, Implementation and Applications. Cambridge University Press, March 2003.

[16] S. Verstichel, B. Volckaert, B. Dhoedt, P. Demeester, and F. De Turck, "Context-Aware Scheduling of Distributed DLReasoning Tasks in Wireless Sensor Networks," International Journal of Distributed Sensor Networks, vol. 2011, 2011.
[17] N. Matthys, C. Huygens, D. Hughes, S. Michiels, and W. Joosen, "A Component and Policy-Based Approach for Efficient Sensor Network Reconfiguration," in Proceedings of IEEE POLICY 2012, Chapel Hill, NC, USA, July 2012.

[18] C. Middleton and A. B. Potter, "Is It Good to Share? A Case Study of the FON and Meraki Approaches to Broadband Provision," in Proceedings of the ITS 17th Biennal Conference, Montreal, Canada, June 2008.

[19] M. Bina and G. M. Giaglis, "Emerging Issues in Researching Community-based WLANs," Journal of Computer Information Systems, vol. 46, no. 1, pp. 9-16, October 2005.

[20] M. Wong and A. Clement, "Sharing Wireless Internet in Urban Neighbourhoods," in Proceedings of the 3rd International Conference on Communities and Technologies (C\&T 2007), East Lansing, MI, USA, June 2007, pp. 275-294.

[21] Meraki, "Cloud Managed Networks that Simply Work," Online, http://www.meraki.com/.

[22] Belgacom, "Wi-Fi Fon Spots with Belgacom," Online, http://www.belgacom.be/onetelco/be-en/catalogue-topic/t_ free_hotspots_res.page.

[23] Telenet, "Telenet Wi-Free," Online, http://telenet.be/3425/nl/ thuis/internet/landingspage/wifree.

[24] H. Velayos and G. Karlsson, "Techniques to Reduce IEEE 802.11b MAC Layer Handover Time," KTH Royal Institute of Technology, Tech. Rep. TRITA-IMIT-LCN R 03:02, April 2003.

[25] S. Shin, A. G. Forte, A. S. Rawat, and H. Schulzrinne, "Reducing MAC Layer Handoff Latency in IEEE 802.11 Wireless LANs," in Proceedings of the 2nd International Workshop on Mobility Management \& Wireless Access Protocols (MobiWac 2004), New York, NY, USA, October 2004, pp. 19-26.

[26] A. Mishra, M.-h. Shin, and W. A. Arbaugh, "Context Caching using Neighbor Graphs for Fast Handoffs in a Wireless Network," in Proceedings of the 23rd Conference of the IEEE Communications Society (IEEE INFOCOM 2004), Hong Kong, March 2004, pp. 351-361.

[27] IEEE P802.11 Task Group R, "IEEE 802.11r Fast Roaming/Fast BSS Transition,” Online, http://grouper.ieee.org/ groups/802/11/Reports/tgr_update.htm.

[28] IEEE P802.11 Task Group K, "IEEE 802.11k Radio Resource Measurement Enhancements," Online, http://grouper.ieee.org/ groups/802/11/Reports/tgk_update.htm.

[29] B. Jooris, A. Schoutteet, F. Vermeulen, and I. Moerman, "Access Network Controlled Fast Handoff for Streaming Multimedia in WLAN," in Proceedings of the 16th IST Mobile and Wireless Communications Summit, Budapest, Hungary, July 2007, pp. 1-5.

[30] R. N. Murty, G. Mainland, I. Rose, A. R. Chowdhury, A. Gosain, J. Bers, and M. Welsh, "CitySense: An Urban-Scale Wireless Sensor Network and Testbed," in Proceedings of the IEEE Conference on Technologies for Homeland Security (HST 2008), Waltham, MA, USA, May 2008, pp. 583-588.

[31] L. Sanchez, J. A. Galache, V. Gutierrez, J. M. Hernandez, J. Bernat, A. Gluhak, and T. Garcia, "SmartSantander: The Meeting Point between Future Internet Research and Experimentation and the Smart Cities," in Proceedings of the Future Network Mobile Summit, Warsaw, Poland, June 2011, pp. 1-8.

[32] Y. Zheng, Y. Liu, J. Yuan, and X. Xie, "Urban Computing with Taxicabs," in Proceedings of the 13th International Conference on Ubiquitous Computing (UbiComp 2011), Beijing, China, September 2011, pp. 89-98. 